\title{
Malaria induced sudden sensorineural hearing loss: a rare presentation
}

\author{
Adewale D. Agbaakin*, Ayodele M. Akinola, Adetola R. Adeyeye, Nonso B. Nkemjika
}

Department of Otorhinolarynology and head and neck surgery, Babcock univerity teaching hospital, Ilisan-Remo, Ogun, Nigeria

Received: 24 July 2019

Revised: 04 October 2019

Accepted: 10 October 2019

\section{*Correspondence:}

Dr. Adewale D. Agbaakin,

E-mail: adewaleagbaakin@gmail.com

Copyright: ( $)$ the author(s), publisher and licensee Medip Academy. This is an open-access article distributed under the terms of the Creative Commons Attribution Non-Commercial License, which permits unrestricted non-commercial use, distribution, and reproduction in any medium, provided the original work is properly cited.

\section{ABSTRACT}

Sudden Hearing loss has been rarely reported as a presenting symptom of malaria fever. It's even more rare to have a bilateral profound sudden sensorineural hearing loss. A 19 years old female student presented with fever, body weakness, hearing loss with tinnitus. Laboratory investigations done were essentially normal except blood film for malaria parasite which showed a high concentration of the parasite in blood on both occasions. Otoacoustics emission test was passed bilaterally while auditory steady state response showed profound hearing loss bilaterally. Normal hearing was restored within $48 \mathrm{hrs}$ of commencement of anti-malaria drugs and low dose steroids.

Keywords: Malaria, Sudden sensorineural hearing loss, Antimalaria, Profound, Otoacoustics emission, Auditory steady state response

\section{INTRODUCTION}

Sudden sensorineural hearing loss (SSNHL) is a sensorineural hearing loss of $30 \mathrm{~dB}$ or more over at least three contiguous audiometric frequencies occurring over 72 hours. The incidence ranges between 5-20 per 100,000 people and most cases have no known cause (Idiopathic). ${ }^{1,2}$ Majority of patients are in their $5^{\text {th }}$ to $6^{\text {th }}$ decade but no gender predilection. Only $2 \%$ of cases have bilateral ear affectation. Tinnitus and dizziness can be associated symptom of SSNHL. ${ }^{1-3}$

A meta-analysis by Chau et al classified the etiological factors for SSNHL into idiopathic $(71 \%)$, infectious (13\%), otologic $(5 \%)$, traumatic $(4 \%)$, vascular $(3 \%)$, neoplastic $(2 \%)$, others $2 \%$ (metabolic, autoimmune and neurologic). ${ }^{4}$ However, parasitic infections among which malaria is one, is not yet a recognised infectious cause of SSNHL.

Malaria is an acute febrile vector transmitted infectious disease of humans caused by parasitic protozoans of
Plasmodium genus: $P$. falciparum, $P$. vivax, $P$. ovale, $P$. Malariae and $P$. Knowlesi. ${ }^{5}$ The disease is characterised by high fever accompanied with shivering, sweating and headache all of which appear after an average of 12 days following a bite by an infective female Anopheles Mosquito. According to World health organization (WHO) spreadsheet report of 2017, P. falciparum is responsible for $99.7 \%$ of malaria in African region and this region alone is responsible $92 \%$ of malaria cases and $93 \%$ of malaria deaths $(435,000 \text { deaths in } 2017)^{5}$.

We present a case of a clinically and laboratory diagnosed malaria induced hearing loss in a patient, with spontaneous recovery after cause specific management was commenced.

\section{CASE REPORT}

A 19 year old student of a private tertiary institution in south-western part of Nigeria presented at the hospital with the following complains; Fever with chills of 24 
hours duration, headaches and Body aches, tinnitus of 3 hours duration and hearing loss of 3 hours duration

She was in her usual state of health until about 24 hours prior to presentation when she noticed she was febrile. It was low grade at onset but progressed over the following hours to high grade. It was intermittent and associated with chills and occasional rigor. There was neither photophobia nor alteration of consciousness.

The headache is frontal and throbbing with pain radiating to the orbit. The generalised Joint and muscle aches made patient uncomfortable but no associated body rashes nor sore throat. Apart from the fever and body aches, patient also had bitter taste and generalised body weakness. Despite these symptoms, patient was able to sleep.

She woke up the following morning with a loud and hash ringing sensation in her ears. The tinnitus was associated with debilitating hearing loss which was sudden, bilateral and disabling as she couldn't hear any sound apart from the ringing in her hear (it was severe enough for attending clinician to differ detailed clerking till patient recovered). The tinnitus was persistent and of same intensity from onset till commencement of treatment. There is associated compensatory high pitched loud voice.

She had an episode of dizziness the same morning while siting from a lying position. The dizziness was described as a feeling of light headedness and was said to have lasted from a few seconds to about 2-3 minutes and no associated nausea and vomiting nor vertigo.

No history of use of neither ototoxic drug nor drug/substance abuse. No preceding history of otologic disease, no exposure to loud noise nor family history of hearing disorder, no history of preceding upper respiratory tract infections. She has no chronic medical illness, genotype is AS. No previous hospital admission. She doesn't smoke nor drink alcohol.
Examination revealed an acutely ill looking patient, conscious and alert, oriented in time place and person, febrile (39.3C), not pale, anicteric, acyanosed, and mildly dehydrated, no lymphadenopathy. Otorhinolaryngology examination revealed an obvious deficit in cranial nerve VIII. Turning fork tests were differed. Other systems yielded no additional information.

Blood film microscopy for malaria showed numerous (>20/hpf) trophozoites of plasmodium falciparum, full blood count was within normal range $\left(9.2 \times 10^{9} / \mathrm{L}\right)$, Erythrocyte sedimentation rate of 14 (using Westergreen tube) normal range 13-16. Brain CT scan showed no brain lesion, however, auditory steady state response (ASSR) showed profound sensorineural hearing loss bilaterally. Otoacoustics emission (OAE) "PASS" bilaterally.

Patient was admitted and rehydrated with normal saline as required, Intramuscular arthemeter at $3.6 \mathrm{mg} / \mathrm{kg}$ stat then $1.8 \mathrm{mg} / \mathrm{kg}$ daily and intravenous dexamethasone at 4 mg 8 hourly were the mainstay of our treatment. Intravenous vitamin B complex in intravenous infusions was administered as an adjunct. And patient closely monitored for late onset neurologic deficit. After about 24 hours of commencing treatment, patient started noticing improvement in her hearing though the tinnitus was persistent but at a lower pitch and loudness. After 48 hours, patient's hearing was back to within normal range (patient's baseline not known) and tinnitus was insignificant.

At discharge, she was placed on oral artemisinin based combination therapy and a daily dose of doxycycline 100 mg for a month.

A repeat hearing evaluation done after patient was discharged showed a mild hearing loss.

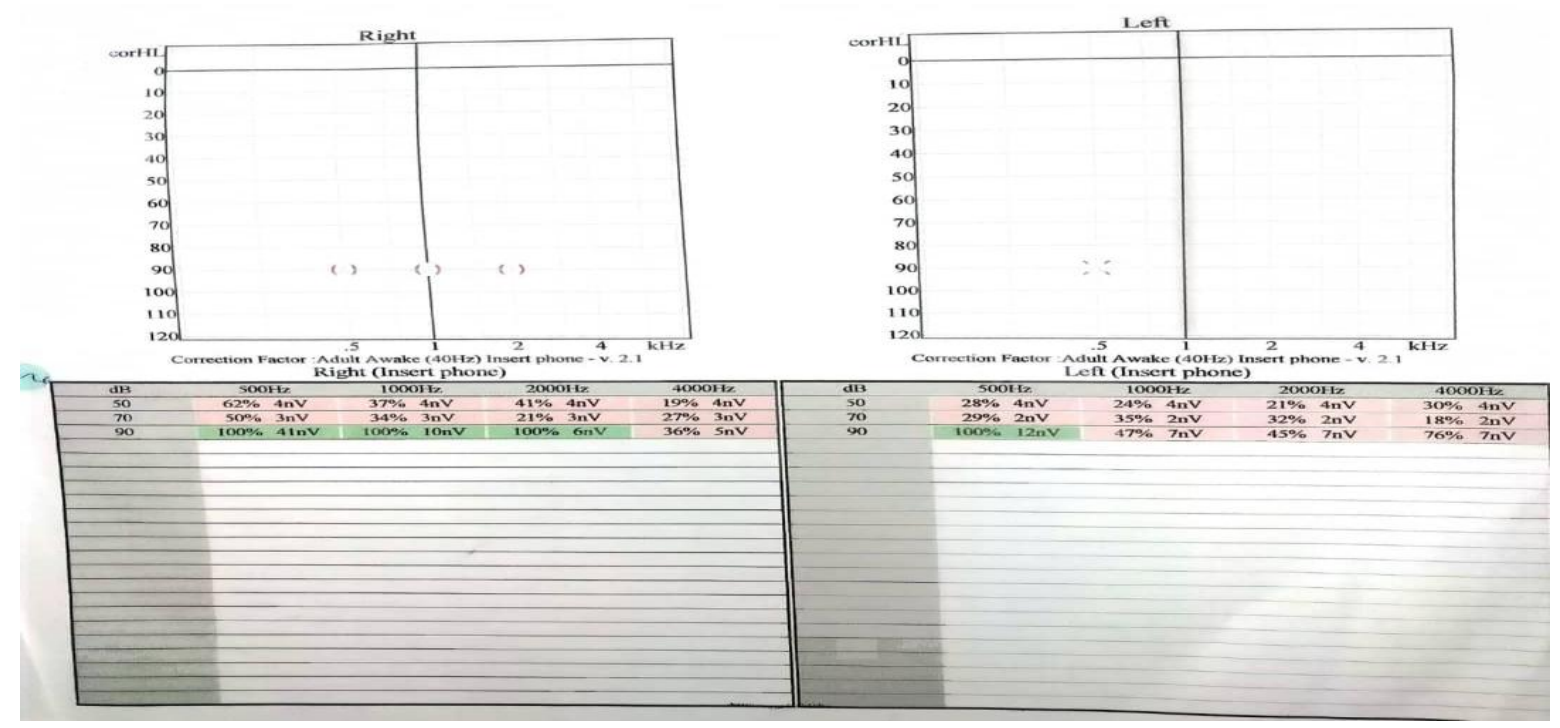

Figure 1: Auditory steady state response report showing profound bilateral hearing loss at presentation. 

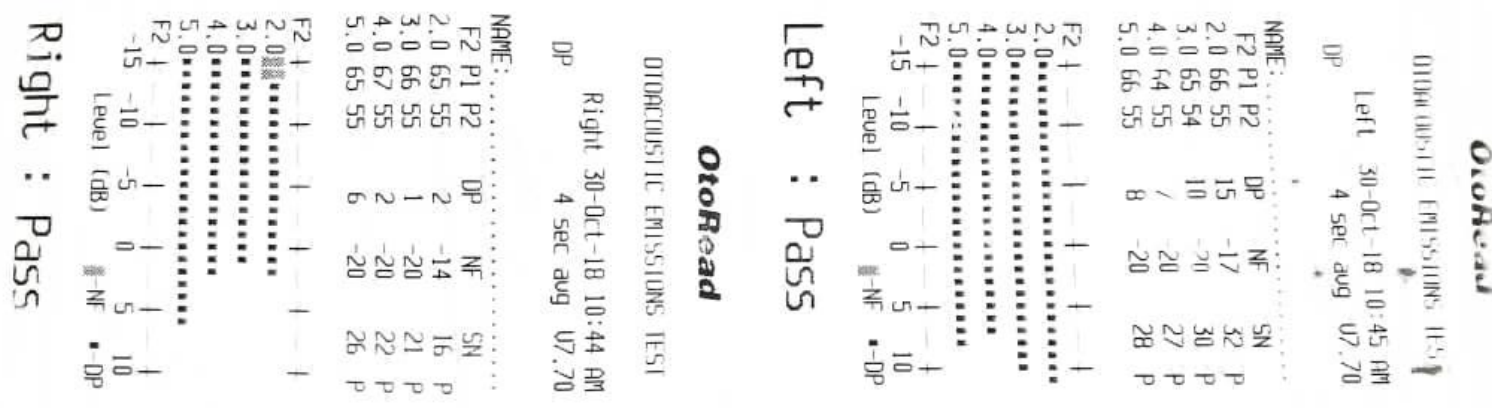

Figure 2: Otoacoustics emission showed 'Pass' bilaterally.

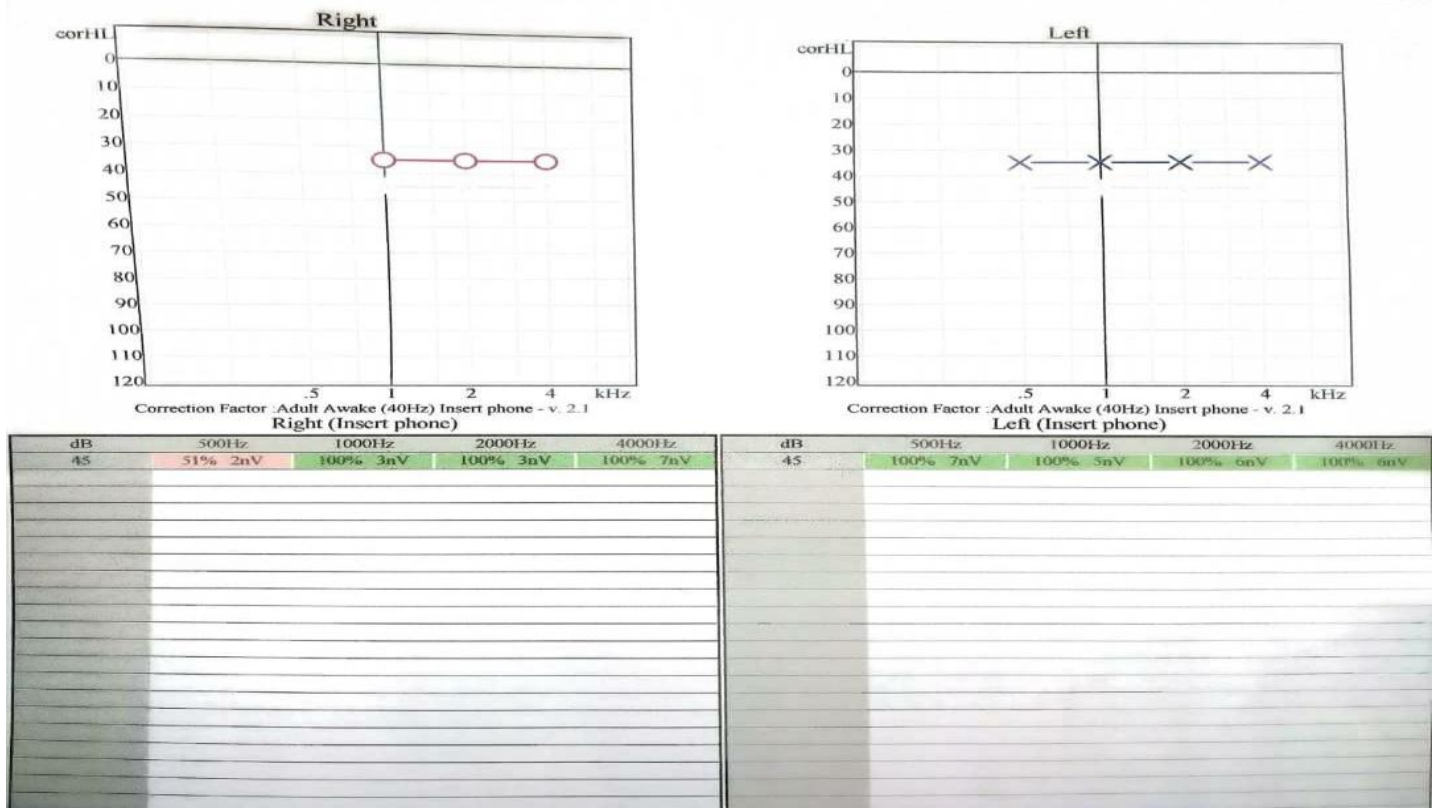

Figure 3: Audiogram showing improvement from profound hearing loss at admission to a mild hearing loss on third day of treatment.

\section{DISCUSSION}

To the best of our knowledge and our online search, we came across a single reported case of malaria induced sudden hearing loss. The case was reported by Tada et al, where they had a case of a 49 year old woman who returned from Burkina Faso with complaints of hearing loss, tinnitus and dizziness followed with fever and altered sensorium. ${ }^{6}$ Definitive diagnosis of severe falciparum malaria was made through blood film microscopy. ${ }^{6}$

The aim of this write up is to corroborate Tada's report by using our experience as an additional proof that hearing loss can be a presenting symptom of malaria rather than just a complication of the disease or its treatment. .

Different theories have been put forward to explain the pathophysiology of sudden sensorineural hearing loss and the most widely accepted ones are vascular theory, cochlear membrane rupture and viral infection theory ${ }^{7}$.
The vascular theory can easily be applied for malaria induced hearing loss through endovascular effect of malaria parasite on the blood supply to either cranial nerves VIII or their cerebral connections.

The blood supply to the cochlea arises from two small terminal arteries. Due to the small diameter of the vessels within the arterial supply and lack of collateral blood supply, the cochlea is susceptible to injury through a variety of vascular insults. The clinical presentation of unilateral sudden SNHL is comparable to the clinical presentation of ischemic vascular diseases such as transient ischemic attacks and amaurosis fugax ${ }^{10}$. The sudden hearing loss due to vascular cause can be as a result of hemmorrage, embolism, vascular diseases, vasospasm or change in blood viscosity.

After erythrocyte invasion, P. falciparum modifies the host cell, changing its morphology and characteristics to cause adherence. The PfEMP-1 ( $P$. Falciparum membrane protein 1) is a key molecule in the definition of the cytoadhesion properties of infected erythrocytes, 
which get close to non-infected erythrocytes to form rosettes, thus allowing binding to several endothelial receptors. Although the invasion of erythrocytes causes the common symptoms of malaria, erythrocyte cytoadhesion is also implicated in the disease's severity. ${ }^{8}$

These theories have shortcomings and cannot fully explain the pathophysiology of sudden sensorineural hearing loss due to malaria.

\section{CONCLUSION}

Hearing loss with or without tinnitus can be a presenting symptom rather than a sequelae of malaria and/or its treatment and these can be treated with antimalaria drugs. The hearing loss/tinnitus could be of varying degree most of which are likely to be subclinical. It is possible that more people with acute malaria have associated sudden hearing loss that is not discovered because the degree of the hearing loss does not significantly affect normal conversation. Hence, a routine audiometric test could be done for all diagnosed cases of malaria to know the prevalence of sudden hearing loss in patients in acute phase of malaria fever.

\section{Funding: No funding sources}

Conflict of interest: None declared

Ethical approval: Not required

\section{REFERENCES}

1. Wilson WR, By FM, Laird N. The efficacy of steroids in the treatment of idiopathic sudden hearing loss. A double-blind clinical study. Acta Oto-Laryngologica. 1980;106:772-6.
2. By FM. Sudden hearing loss: eight years' experience and suggested prognostic table. Laryngoscope. 1998;94: 647-661

3. Fetterman BL, Saunders JE, Luxford WM. Prognosis and treatment of sudden sensorineural hearing loss. Am J Otol. 1996;17:529-36.

4. Chau JK, Lin JR, Atashband S, Irvine RA, Westerberg BD. Systematic review of the evidence for the etiology of adult sudden sensorineural hearing loss. Laryngoscope. 2010;120:1011-21.

5. World Health Organization. Guidelines for the Treatment of Malaria. 3rd ed. Geneva: World Health Organization; 2015. Available from: http://www.who.int/malaria/publications/atoz/97892 41549127/en/. Accessed on 18th July 2019.

6. Tada T, Hitani A, Honda NH, Haruna S, Yoshimura T, Haruki K, et al. A case of falciparum malaria: Acute hearing loss as the initial symptom. J Infect Chemother. 2017;23(1):56-8.

7. Kuhn M, Heman-Ackah SE, Shaikh JA, Roehm PC. Sudden Sensorineural Hearing Loss: A Review of Diagnosis, Treatment, and Prognosis. Trends Hearing. 2011;15(3):91-105.

8. Pasternak ND, Dzikowski R. PfEMP1: an antigen that plays a key role in the pathogenicity and immune evasion of the malaria parasite Plasmodium falciparum. Int $\mathbf{J}$ Biochem Cell Biol. 2009;41(7):1463-6.

Cite this article as: Agbaakin AD, Akinola AM, Adeyeye AR, Nkemjika NB. Malaria induced sudden sensorineural hearing loss: a rare presentation. Int J Otorhinolaryngol Head Neck Surg 2019;5:1701-4. 